\title{
Comparison of Fuzzy Contrast Enhancement Techniques
}

\author{
G.Sudhavani \\ Associate Professor, \\ Department of ECE, \\ RVR \& JC College of \\ Engineering, Guntur.
}

\author{
M.Srilakshmi \\ Student (M.Tech), \\ Department of ECE, \\ RVR \& JC College of \\ Engineering, Guntur.
}

\author{
P.Venkateswara Rao \\ Associate Professor, \\ Department of CSE, \\ RVR \& JC College of \\ Engineering, Guntur.
}

\author{
K.Satya Prasad \\ Professor, \\ Department of ECE, \\ JNTU Kakinada, Kakinada.
}

\begin{abstract}
The aim of the image enhancement is to improve the interpretability or perception of the information in images for human viewers, or to provide 'better' input for other automated image processing techniques. It is an indispensable tool for researchers in wide verity of fields including art studies, medical imaging, forensics and atmospheric sciences. Most of images like satellite images, medical images and even real life photographs may suffer from poor contrast due to the inadequate or insufficient lighting during image acquiring. So it is necessary to enhance the contrast of an image. In this paper two enhancement techniques namely fuzzy rule based contrast enhancement, and contrast enhancement using intensification operator (INT) are presented for the low contrast grayscale images. In first technique fuzzy system response function is obtained by simple if-then rules, and in second technique the fuzzy contrast intensification operator is taken as a tool for the enhancement in the fuzzy property domain. Comparative analysis of these enhancement techniques is carried out by means of index of fuzziness (IOF) and processing time.
\end{abstract}

\section{Keywords}

Image, enhancement, contrast enhancement, fuzzy logic, membership function, intensification operator, fuzzy expected value, fuzzifiers, index of fuzziness, processing time.

\section{INTRODUCTION}

In digital image processing enhancement is a fundamental task to improve interpretability and appearance of an image and is applicable in every field where images are to be understood and analyzed. Image enhancement includes a variety of operations such as noise removal [1] [2], deblurring, and gray-level dynamic range modification. Contrast enhancement is one of the enhancement operations and is often part of image processing systems [3] in the pre processing and / or post-processing stage. If the intensity levels of the pixel reside densely in a narrow range in the histogram of the image, then it is said to be a low contrast image. The objects which lie in this type of image are not distinct. To improve the quality of the image and visual perception of human beings, different number of enhancement methods are to be applied [4],[5].
Good contrast images with preserving details are required for many important areas such as remote sensing, machine vision, biomedical image analysis, dynamic and traffic sense analysis, and autonomous navigation. However most of the recorded images suffer from low contrast, which is due to insufficient lighting during image acquiring, wrong setting of shutter speed and aperture size. Thus contrast enhancement is employed to increase contrast of the image. Enhancement of the images is also useful in edge detection. A good contrast image produces better result for edge detection. An edge detector produces either a set of edge points or edge fragments. Edge detection provides a means of segmenting [6] [7] the image into meaningful regions.

Image enhancement techniques can be divided into two categories namely spatial domain and frequency domain methods [8]. The spatial domain method refers to aggregate of pixels composing an image, and they operate directly on the pixels of image. The frequency domain method refers to an aggregate of complex pixels resulting from taking the Fourier Transform and arises from the fact that this particular transform is composed of complex sinusoids. Due to the vast processing requirements, frequency-domain techniques are not widely used as spatial domain techniques. However, the enhancement in frequency domain is time consuming process even with fast transformation technique, thus made it unsuitable for real time applications [9].

In this paper fuzzy techniques are used for the enhancement of images. There are many reasons for using fuzzy techniques in image processing, those are as follows. In many image processing applications, expert knowledge is required to overcome the difficulties (like object recognition, scene analysis). Fuzzy set and fuzzy logic [10] offers us powerful tool to represent and process human knowledge in form of fuzzy if-then rules. Because of the data uncertainty due to randomness, ambiguity and vagueness many difficulties arise in image processing. Fuzzy method can manage ambiguity and vagueness efficiently.

This paper presents two enhancement techniques based on the fuzzy logic to increase the contrast of structures of interest in an image. In first technique the fuzzy system response function is acquired by simple fuzzy if then rules. In second 
technique the fuzzy contrast intensification operator is considered as a means for the enhancement.

\section{FUZZY CONTRAST ENHANCEMENT TECHNIQUES}

Contrast of image is measure of difference between the graylevels in an image. Higher the contrast, greater is the distinction between gray-levels in the image. High contrast images have either all black or all white regions; there are very little similar gray-levels in the image, and very few white or black regions. They can be thought of as crisp and low contrast ones as completely fuzzy. The images with good standard of grays between black and white are usually the best images for purposes of recognition by humans.

The objective of contrast enhancement is to process a given image so that the result is more suitable than the original image for any application. In the entire image processing techniques special care has to be taken, so that the processed image is not distinctly different from the original image. The technique used here makes use of modifications to brightness membership value in stretching or contracting the contrast of an image.

An image $\mathrm{G}$ of $\mathrm{M} \times \mathrm{N}$ dimensions is considered as an array of fuzzy singletons, each having a membership value denoting the degree of brightness level p, $p=0,1,2 \ldots$ P-1 (e.g., range of densities from $p=0$ to $p=255$ ), or some relative pixel density. By using the notation of fuzzy sets, an image can be represented as follows,

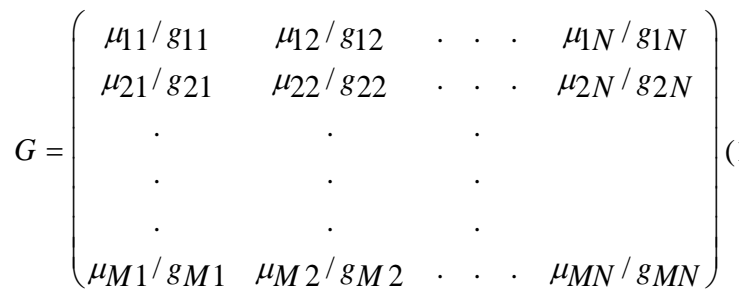

Where $0 \leq \mu_{m n} \leq 1, \mathrm{~m}=1,2 \ldots \mathrm{M}, \mathrm{n}=1,2 \ldots \mathrm{N}$.

In this paper, two fuzzy contrast enhancement techniques are investigated and their performance is compared by some measures of fuzziness.

\subsection{Fuzzy Rule Based Contrast Enhancement}

Gray scale transformations, with the image contrast enhancement as a main application, are among the most frequent areas in which fuzzy techniques for image processing are applied [11] [12]. To express the image contrast enhancement in terms of fuzzy logic, Takagi - Sugeno fuzzy rule based system [11] [12] is used. This rule based approach includes the following steps.

Step 1: Specifying the input membership functions.

Step 2: Specifying the output membership functions.

Step 3: Obtaining the fuzzy system response function $F$ of following rules.

$$
\begin{aligned}
& \text { IF a pixel is dark, THEN make it darker } \\
& \text { IF a pixel is gray, THEN make it gray } \\
& \text { IF a pixel is bright, THEN make it brighter }
\end{aligned}
$$

Step 4: Construct intensity transformation function $\mathrm{T}$ using fuzzy system response function $\mathrm{F}$.

Step 5: Transform the intensities of input image using $T$.

A common formulation as imagines the interpretation of the gray scale of the input image by three linguistic terms, denoted by dark, gray and bright. The terms dark and bright are represented by trapezoidal-shaped fuzzy membership functions. The term gray is described by a triangular shaped membership function as illustrated in Fig 1 .

With reference to output, making intensities darker and brighter increases the separation of dark and light on the gray scale, which in turn increases the contrast. Usually, narrowing the mid grays increases the "richness" of the image. Fig-2 represents a set of output membership functions plot which uses the three bell shaped membership functions for three variables darker, midgray and brighter.

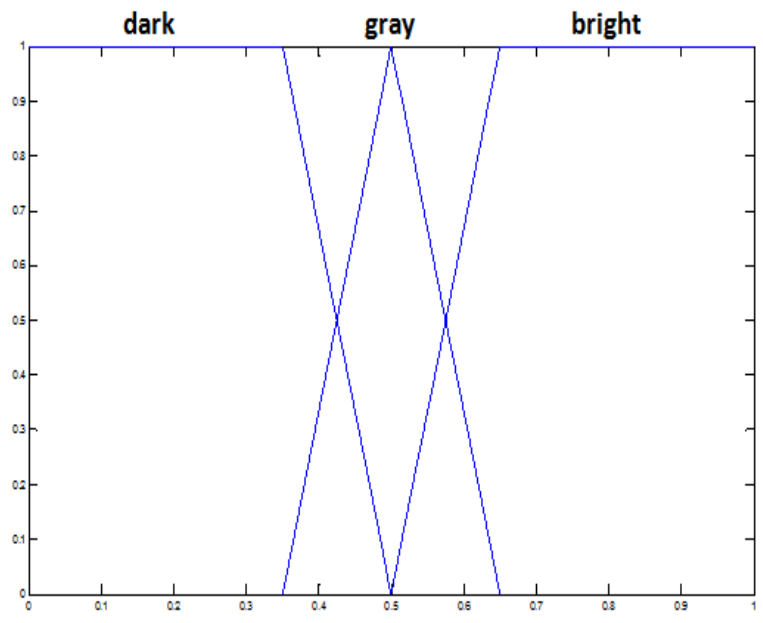

Fig 1: Input membership functions for fuzzy rule based contrast enhancement

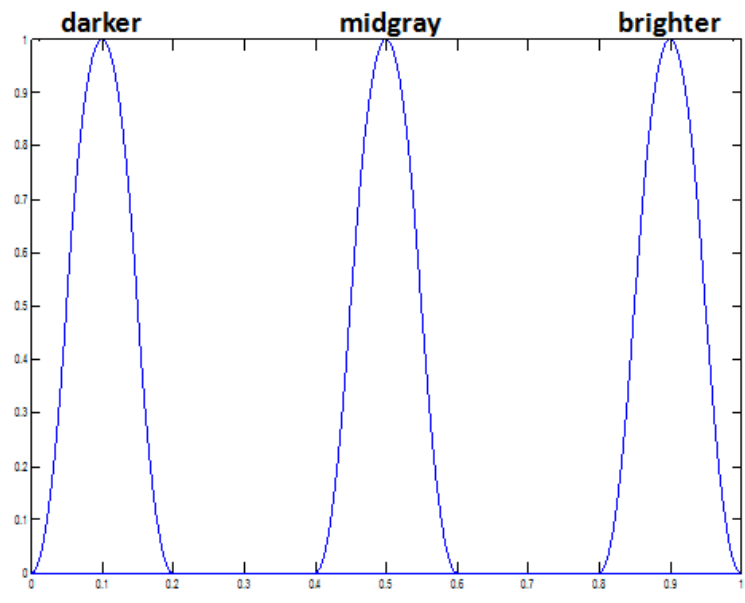

Fig 2: Output membership functions for fuzzy rule based contrast enhancement

\subsection{Contrast Enhancement Using Fuzzy INT-Operator}

This technique uses the intensification operator [13] to decrease the fuzziness of the image which results in an increase of contrast of image [14] [15]. The algorithm can be formulated as follows:

Step 1: Defining the parameters of membership function in Eq. (2)

Step 2: Fuzzification of the gray levels by using the following membership function.

$\mu_{m n}=G\left(g_{m n}\right)\left[1+\frac{g_{\max }-g_{m n}}{F_{d}}\right]^{-F_{e}}$ 
Where

$$
\begin{aligned}
& F_{e} \text { - Exponential fuzzifiers } \\
& F_{d} \text { - Denomination fuzzifiers } \\
& g_{m i d} \text { - Mid gray level } \\
& g_{\max } \text { - Maximum gray level (L-1) desired } \\
& g_{m n} \text { - Intensity value of }(\mathrm{m}, \mathrm{n}) \text { pixel of input image }
\end{aligned}
$$

$F_{d}$ is calculated by using the below expression

$$
F_{d=} \frac{g_{\max }-g_{\text {mid }}}{0.5 \overline{F_{e}}-1}
$$

Step 3: Membership values are modified by the following transformation.

$$
\mu_{m n}^{\prime}=\left\{\begin{array}{cc}
2 \cdot\left[\mu_{m n}\right]^{2} & 0 \leq \mu_{m n} \leq 0.5 \\
1-2 \cdot\left[1-\mu_{m n}\right]^{2} & 0.5 \leq \mu_{m n} \leq 1
\end{array}\right\}
$$

Step 4: New gray levels $g_{m n}^{\prime}$ are generated using $G^{-1}$.

$$
g_{m n}^{\prime}=G^{-1}\left(\mu_{m n}^{\prime}\right)=g_{\max }-F_{d}\left(\left(\mu_{m n}^{\prime}\right)^{-1 / F_{e}}-1\right)
$$

Applying the contrast intensification operator on an image $G$ creates another image $G^{\prime}=I N T(G)$ in which the fuzziness is decreased by increasing the $\mu_{m n}$ values that are greater than 0.5 and decreasing the values that are less than 0.5 as shown in Eq.(4).

Example: Consider an array of 25 pixels as shown below

Table 1. Array of pixels with given intensities

\begin{tabular}{|l|l|l|l|l|}
\hline 110 & 105 & 140 & 117 & 110 \\
\hline 110 & 132 & 105 & 115 & 154 \\
\hline 140 & 105 & 105 & 115 & 154 \\
\hline 137 & 105 & 145 & 150 & 150 \\
\hline 140 & 118 & 115 & 109 & 148 \\
\hline
\end{tabular}

In the next step the above pixel values are scaled to obtain the membership functions of each of the pixel using Eq. (2) and are shown in Table 2.

Table 2. Scaled values indicating memberships of each pixel

\begin{tabular}{|c|c|c|c|c|}
\hline 0.43 & 0.41 & 0.55 & 0.42 & 0.43 \\
\hline 0.43 & 0.52 & 0.43 & 0.59 & 0.41 \\
\hline 0.55 & 0.41 & 0.41 & 0.45 & 0.60 \\
\hline 0.54 & 0.53 & 0.57 & 0.59 & 0.59 \\
\hline 0.55 & 0.46 & 0.45 & 0.42 & 0.58 \\
\hline
\end{tabular}

Then the membership values are modified by the transformation in Eq.4 for contrast enhancement of the array of pixels.
If the pixel of intensity is 0.43 , then the new intensity value is, $2 \times 0.43^{2}=0.37$ as $0<0.43<0.5$.

If the pixel of intensity is 0.55 , then its new intensity value is $\left[1-2(1-0.55)^{2}\right]=0.60$

In this manner all the new intensities of the remaining pixels are calculated and shown in Table 3

Table 3. Membership values of the pixels after application of INT operator

\begin{tabular}{|c|c|c|c|c|}
\hline 0.37 & 0.33 & 0.60 & 0.35 & 0.37 \\
\hline 0.37 & 0.54 & 0.37 & 0.66 & 0.33 \\
\hline 0.60 & 0.33 & 0.33 & 0.40 & 0.68 \\
\hline 0.57 & 0.56 & 0.63 & 0.66 & 0.66 \\
\hline 0.60 & 0.42 & 0.40 & 0.35 & 0.55 \\
\hline
\end{tabular}

Thus the Table 3 shows that the pixels having the membership values greater than 0.5 have been increased in intensity and those with value less than 0.5 have been decreased in intensity,

\section{SIMULATION RESULTS}

In this paper two techniques for fuzzy contrast enhancement have been implemented. To measure the quality of the original and enhanced images, the linear index of fuzziness $\gamma$ is considered and is expressed below in the Eq. (6)

$$
\gamma=\frac{2}{M N} \sum_{M} \sum_{N} \min \left(\mu_{m n}, 1-\mu_{m n}\right)
$$

The index of fuzziness was defined by Kaufmann [16]. It gives the ambiguity in an image by calculating the distance between its fuzzy property plane and the nearest ordinary plane [17]. Global grayness ambiguity (fuzziness) of an image is calculated by the index if fuzziness. They can be considered as degree of difficulty in deciding whether a pixel would be processed as white (bright) or black (dark) [18]. Processing time is taken as another tool for performance measure. Table 4 and 5 shows the measure of fuzziness and processing time to ascertain the performance of the proposed two fuzzy contrast enhancement algorithms. Fig 3 and Fig 4 shows the graphical representation of the index of fuzziness and processing time for two fuzzy contrast enhancement algorithms. Fig 5 to 9 represents the results of fuzzy rule based contrast enhancement and contrast enhancement using INT operator for five low contrast grayscale images. 
Table 4. Measure of index of fuzziness to ascertain performance of fuzzy rule based contrast enhancement and contrast enhancement using INT operator

\begin{tabular}{|c|c|c|c|}
\hline \multicolumn{5}{|c|}{ Index of Fuzziness $\gamma$} \\
\hline Image & $\begin{array}{c}\text { Input } \\
\text { Image }\end{array}$ & $\begin{array}{c}\text { Output of } \\
\text { rule based } \\
\text { method }\end{array}$ & $\begin{array}{l}\text { Output of } \\
\text { INT operator } \\
\text { method }\end{array}$ \\
\hline Satellite & 0.4397 & 0.4002 & 0.3726 \\
\hline Cells & 0.4216 & 0.4072 & 0.3854 \\
\hline Rose & 0.4379 & 0.3605 & 0.3347 \\
\hline Buildings & 0.4191 & 0.3358 & 0.3072 \\
\hline Whale & 0.5804 & 0.5011 & 0.4591 \\
\hline
\end{tabular}

Table 5. Measure of processing time to ascertain performance of fuzzy rule based contrast enhancement and contrast enhancement using INT operator

\begin{tabular}{|c|c|c|}
\hline \multicolumn{3}{|c|}{ Processing Time (sec) } \\
\hline Image & $\begin{array}{c}\text { Fuzzy rule } \\
\text { based method }\end{array}$ & $\begin{array}{c}\text { INT operator } \\
\text { method }\end{array}$ \\
\hline Satellite & 4.2514 & 8.3392 \\
\hline Cells & 2.1367 & 4.8772 \\
\hline Rose & 4.5458 & 8.4505 \\
\hline Buildings & 14.9405 & 22.2671 \\
\hline Whale & 3.2142 & 6.6215 \\
\hline
\end{tabular}

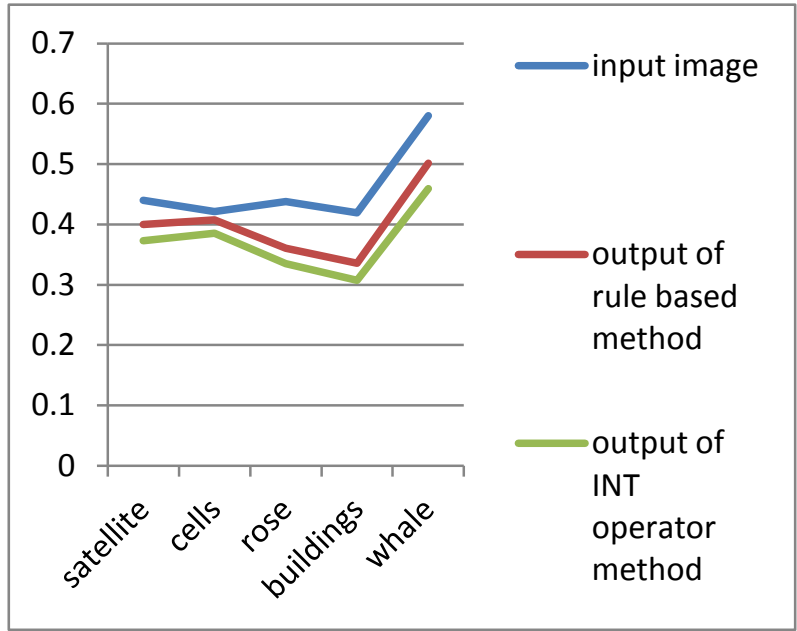

Fig 3: Graphical representation of index of fuzziness for two fuzzy contrast enhancement algorithms

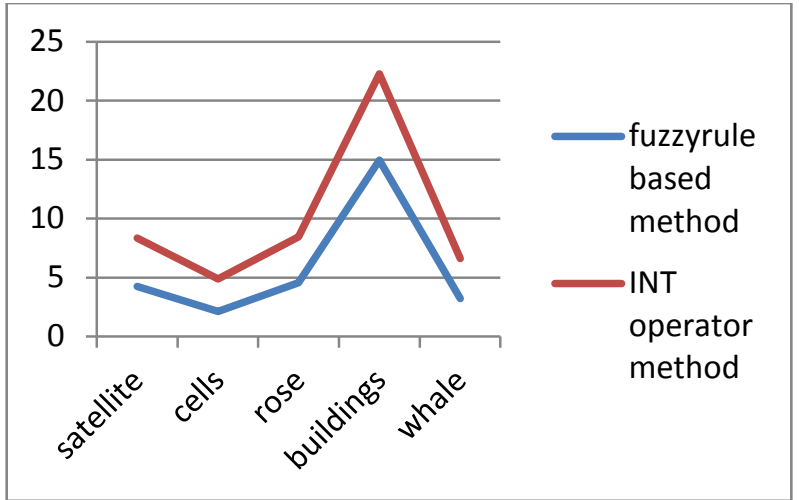

Fig 4: Graphical representation of processing time for two fuzzy contrast enhancement algorithms

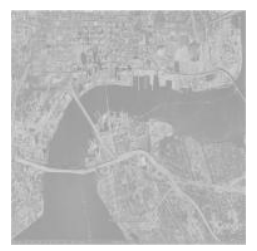

(a)

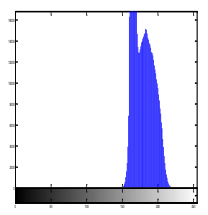

(d)

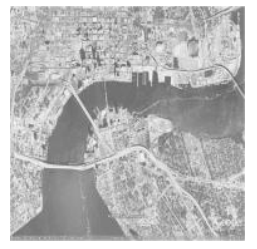

(b)

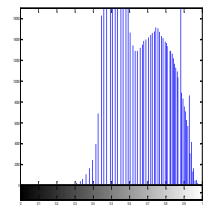

(e)

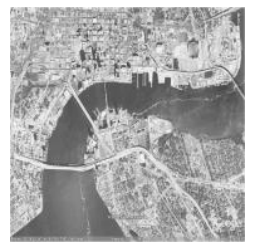

(c)

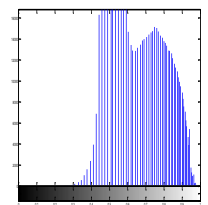

(f)
Fig 5: Illustration of two algorithms for Satellite image, a) Original image, b) output of fuzzy rule based method, c) output of fuzzy INT operator method, d) histogram of (a), e) histogram of (b), f) histogram of (c).

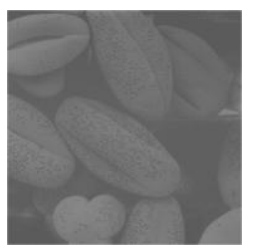

(a)

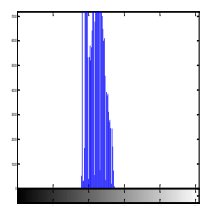

(d)

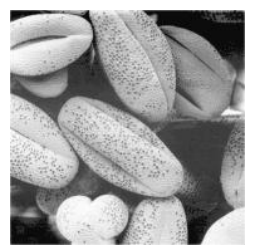

(b)

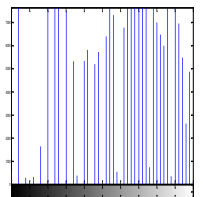

(e)

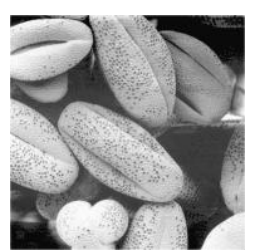

(c)

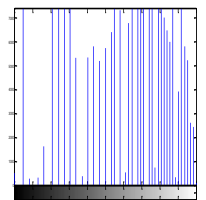

(f)
Fig 6: Illustration of two algorithms for Cells image, a) Original image, b) output of fuzzy rule based method, c) output of fuzzy INT operator method, d) histogram of (a), e) histogram of (b), f) histogram of (c). 


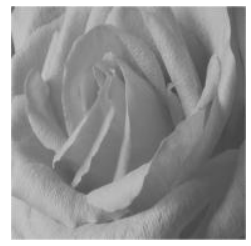

(a)

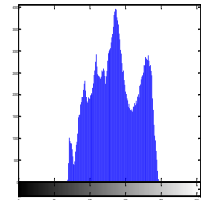

(d)

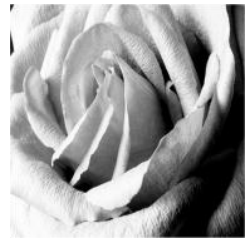

(b)

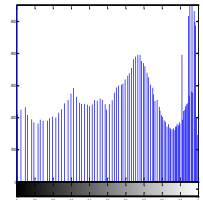

(e)

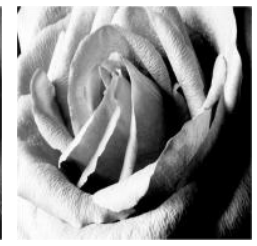

(c)

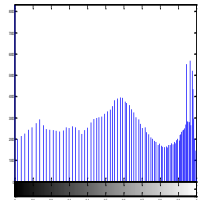

(f)
Fig 7: Illustration of two algorithms for Rose image, a) Original image, b) output of fuzzy rule based method, c) output of fuzzy INT operator method, d) histogram of (a), e) histogram of (b), f) histogram of (c).

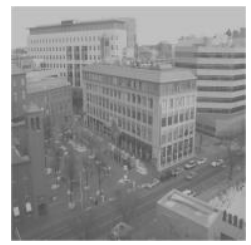

(a)

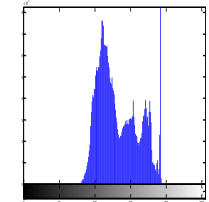

(d)

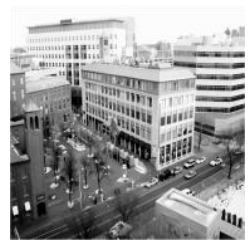

(b)

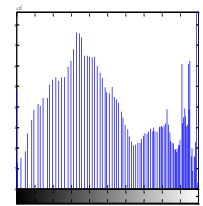

(e)

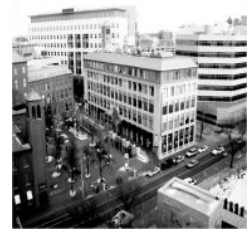

(c)

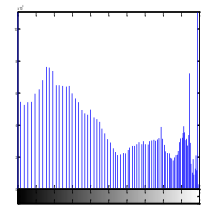

(f)
Fig 8: Illustration of two algorithms for Buildings image, a) Original image, b) output of fuzzy rule based method, c) output of fuzzy INT operator method, d) histogram of (a), e) histogram of (b), f) histogram of (c).

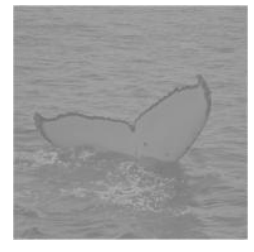

(a)

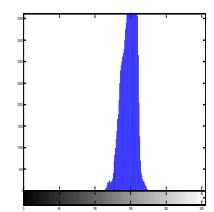

(d)

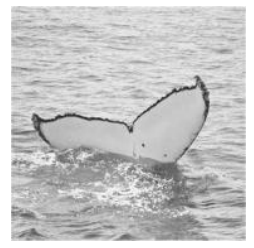

(b)

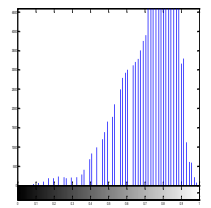

(e)

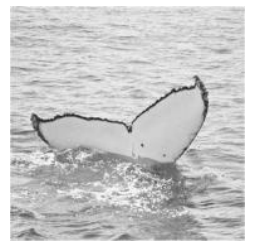

(c)

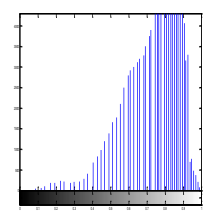

(f)
Fig 9: Illustration of two algorithms for Whale image, a) Original image, b) output of fuzzy rule based method, c) output of fuzzy INT operator method, d) histogram of (a), e) histogram of (b), f) histogram of (c).

\section{CONCLUSION}

In this paper, two different fuzzy approaches have been implemented for the enhancement of low contrast grayscale images. Compared with classical approaches, it is observed that, the fuzzy methods offer a powerful mathematical framework for developing new enhancement algorithms that allows the use of expert knowledge in processing steps.

From the visual observations (Fig 5 to Fig 9), it is clear that there is an increase in the contrast of the images. It is also observed that the histograms of original image \& enhanced images have the same basic characteristics, which is not possible in histogram equalization.

The subjective quality of the output of the two implemented algorithms is satisfactory. In this paper two parameters used for comparing the two techniques namely, index of fuzziness and processing time. A lower value of index of fuzziness indicates a good enhanced image. From Table 4 it can be observed that, the contrast enhancement using INT operator technique has lower values of index of fuzziness when compared to the fuzzy rule based contrast enhancement technique and is shown graphically in Fig 3. In the case of processing time, fuzzy rule based contrast enhancement technique requires less time than the contrast enhancement using INT operator technique and is shown graphically in Fig 4.

\section{REFERENCES}

[1] G. Sudhavani, G.Madhuri, P. Venkateswara Rao \& K. Satya Prasad "Removing of Gaussian Noise from Color Images by Varying Size of Fuzzy Filter", International Journal of Computer applications (09751 - 8887), vol. 72, No. 17, June 2013.

[2] G. Sudhavani, G. Madhuri, P. Venkateswara Tao \& Dr. K. Satya Prasad "ADDITIVE NOISE REMOVAL FOR COLOR IMAGES USING FUZZY FILTERS", International Journal of Computer Science, Engineering Applications (IJCSEA) vol. 3, No. 3, June 2013.

[3] Etiene E. Kerre and Mike Nachtegael "Fuzzy Techniques in Image Processing" Studies in fuzziness and soft computing, ISSN 1434-9922, 2000.

[4] Byoung-Woo Yoon, Woo-Jin Song "Image contrast enhancement based on the generalized histogram" Journal of Electronic Imaging 16(3), 033005 (July - Sep 2007).

[5] Dr. H. Mamatha Devi, S. Somorjeet Singh, Th. Tangkeshwar Singh, O. Imocha Singh "A New Easy Method of Enhancement of Low Contrast Image using Spatial Domain" International Journal of Computer Applications (0975 - 8887) vol. 40, No. 1, February 2012.

[6] G. Sudhavani \& Dr. K.Satya Prasad "Segmentation of Lip image by Modified Fuzzy C-means Clustering Algorithm" International Journal of Computer Science \& Network Security, vol. 9, No. 4, April 2009.

[7] G. Sudhavani, S. Sravani, P. Venkateswara Rao \& K. Satya Prasad "Fuzzy Edge Linking Process on Fuzzy Noise Filtered Image", International Journal of Computer Applications (0975-8887), vol. 93, No. 15, May 2014.

[8] Raman Maini and Himanshu Aggarwa "A Comprehensive Review of Image Enhancement 
Techniques" JOURNAL OF COMPUTING VOLUME 2, ISSIE 3, MARCH 2010, ISSN 2151 - 9167.

[9] Khairunnisa Hasikin \& Nor Ashidi Mat Isa "Enhancement of the low contrast image using fuzzy set theory", $14^{\text {th }}$ International Conference on Modeling and Simulation, IEEE, 2012.

[10] E. E. Kerre, Fuzzy sets and Approximate Reasoning, Xian, China: Xian Jiaotong Univ. Press, 1998.

[11] R. C. Gonzalez, R. E. Woods, Digital Image Processing", $3^{\text {rd }}$ Ed., Prentice Hall,

[12] W. Pedrycz, F. Gomode "An Introduction to Fuzzy Sets: Analysis and Design", the MIT Press, 1998.

[13] ZADEH. L. A, "Fuzzy-Set-Theoretic Interpretation of Linguistic Hedges”, J. Cybern., vol. 2, pp. 4-34, 1972.

[14] SANKAR K. PAL, ROBERT A. KING, "Image Enhancement using Smoothing with Fuzzy Sets", IEEE
Transactions on Systems, Man and Cybernetics, vol. SMC-11, No. 1, pp. 494-501, July 1981.

[15] SANKAR K. PAL, ROBERT A. KING, "On edge detection of X-ray images using fuzzy sets", IEEE Transactions on pattern Analysis and Machine Intelligence, vol. PAMI-5, No. 1, pp. 69-77, 1983.

[16] KAUFMANN, A., "Introduction to the Theory of Fuzzy Sub-Sets-Fundamental Theoretical Elements", vol. 1, Academic Press, New York, 1975.

[17] SANKAR K. PAL, "Fuzziness, Image Information and Scene Analysis", in: Zadeh. L. A, Yager. R. R, "An Introduction to Fuzzy Logic Applications in Intelligent Systems", Kluwer Academic Publishers, USA, 1996.

[18] SANKAR K. PAL, KUNDU, M. K, "Automatic selection of object enhancement operator with quantitative justification based on fuzzy set theoretic measures, Pattern Recognition Letters", vol. 11, pp. 811829, 1990. 